\title{
MOLINO SAVONIUS. PROYECTO DE EXTENSIÓN Y MARCO DIDÁCTICO EN CLASES DE MATEMÁTICA
}

Dr. Pablo Carranza (*)

\section{RESUMEN}

En la presente comunicación compartiremos algunas notas referidas a un trabajo llevado a cabo durante el primer semestre del año 2015 en el marco de las clases de matemática de la Tecnicatura en Mantenimiento Industrial de la Universidad Nacional de Río Negro. Este trabajo realizado con los estudiantes de primer año tomó la forma institucional de un proyecto de extensión, al mismo tiempo que para nosotros representó un marco didáctico y pedagógico para el desarrollo de nuestras clases de matemática. Se trató del cálculo y construcción de un molino tipo Savonius para ser instalado en la vivienda de una pobladora rural del norte de la Patagonia. Comenzaremos describiendo el lugar donde se instaló el molino.

\section{Contexto sociogeográfico}

El molino tipo Savonius construido con los estudiantes fue instalado en un puesto rural ubicado a unos $50 \mathrm{kms}$ al norte del lago Pellegrini, provincia de Río Negro, Argentina. La geografía de la zona en cuestión podría describirse como de meseta semidesértica, coma la mayor parte de la Patagonia Argentina por cierto. Aquí, las precipitaciones no superan los $200 \mathrm{~mm}$ anuales, la vegetación es escasa y no solo por las raras lluvias sino también por los fuertes vientos predominantes del oeste.

Los pobladores que habitan en la zona de meseta, suelen tener una economía de subsistencia basada en la cría extensiva de algunos caprinos y/u ovinos. La disponibilidad de agua es determinante para la ubicación de sus puestos (casas en el campo). Ellos suelen localizarse a orillas de vertientes o como es nuestro caso, donde ha sido posible encontrar agua en pozos llamados jagüeles.

Nuestro molino fue construido para una pobladora rural de esta zona de meseta llamada Yolanda, una mujer de aproximadamente unos 60 años que vive sola en su puesto, juntos a sus animales. En el puesto, Yolanda cuenta con dos jagüeles, ninguno de ellos tiene agua apta para el consumo humano pero sí para bebida de sus animales y riego de sus árboles. El agua de estos jagüeles era extraía por Yolanda a mano por medio de una bomba tipo sapo. Nos propusimos con los estudiantes construir un molino para la extracción de agua y así facilitarle las tareas a esta pobladora. Describiremos brevemente el tipo de molino construido.

\section{Molino savonius}

El molino de tipo Savonius es un rotor 
de eje vertical. Se le reconocen al menos dos grandes virtudes a este diseño, por un lado su facilidad constructiva, por el otro su economía en términos de piezas a fabricar. Una de sus principales desventajas reside en el bajo rendimiento, producto del giro de una de sus paletas en contra del viento.

Sin embargo y dados los fuertes vientos reinantes en la zona, optamos por construir un molino del tipo Savonius. La figura 1 muestra una ilustración de este tipo de molinos donde el rotor está constituido por medios tambores dispuestos verticalmente $\mathrm{y}$ un eje que relaciona el rotor con una bomba tipo diafragma ubicada al pie del molino. La bomba a diafragma es la encargada de la extracción del agua del jagüel.

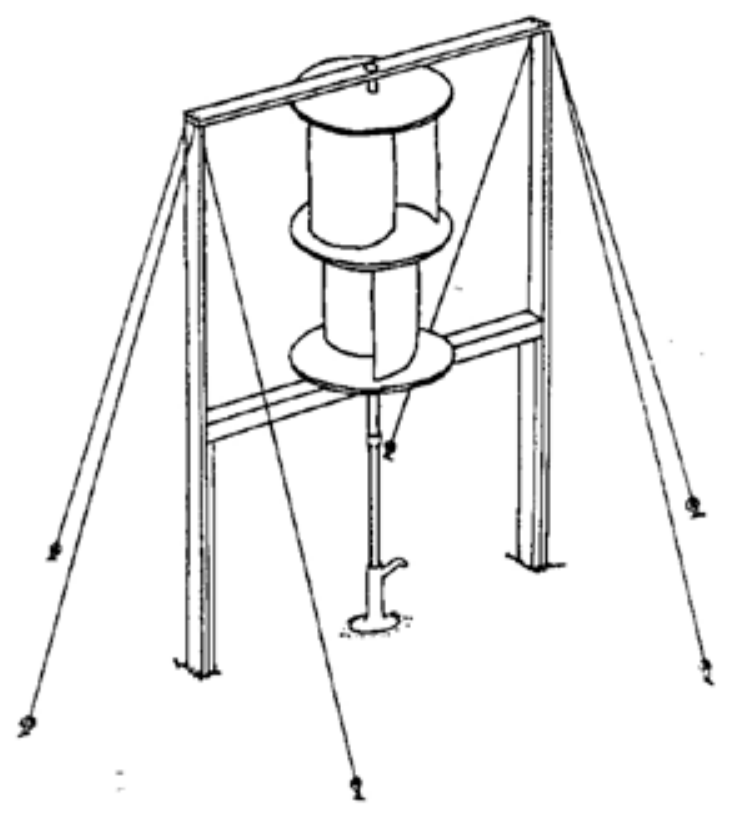

Figura 1. Esquema del molino tipo Savonius

Presentaremos brevemente las motivaciones que guiaron este proyecto.

\section{MOTIVACIONES}

El proyecto tuvo varios objetivos interrelacionandos, todos de connotaciones didácticas. Presentaremos algunos de ellos bajo la forma de motivaciones.

\section{La función social del conocimiento}

Entendemos que los saberes organizados bajo la forma de disciplinas son una producción cultural. Entendemos también que esta producción cultural puede (y debe intentarse) ser devuelta a la comunidad mediante acciones que sean de beneficio directo y colectivo. También entendemos que esta devolución es factible de realizarse en el proceso de aprendizaje de los estudiantes, quienes en nuestro caso, y si consideramos sus diferentes procesos de aprendizajes institucionalizados tales como la escuela primaria y la secundaria, llevan al menos 12 años aprendiendo conceptos disciplinares.

En nuestro caso, se consideraron sa- 
beres de disciplinas tales como Matemática, Física y Estadística. Así, los conocimientos adquiridos anteriormente y los en proceso en el marco de la Tecnicatura en Mantenimiento Industrial sirvieron a los estudiantes para construir e instalar el molino en el puesto de Yolanda.

\section{La integración de disciplinas}

También entendemos que en la vida real, tanto sea profesional como personal, raras son las situaciones donde se utilizan conceptos de una sola disciplina. Bien al contrario, consideramos que la mayoría de las situaciones a las que nos enfrentamos convocan o deberían convocar a varias de ellas para así optimizar su solución.

Por ello, en este proyecto nos interesamos a su integración y tratamiento conjunto. En efecto, para la construcción e instalación del molino fueron necesarios conceptos y métodos de disciplinas varias. Reconocemos que y por diferentes razones, algunas de ellas no pudieron ser convocadas, tal es el caso de la sociología por ejemplo.

Los conocimientos como argumentos para las tomas de decisiones

En nuestra propuesta, el esquema de funcionamiento pretendido para los saberes adquiridos y los en proceso de adquisición podría describirse como el de un conjunto de argumentos para las tomas de decisiones que el proyecto requería.

En efecto, para nuestro trabajo no se contó con planos acabados listos para ser utilizados como referencia para la construcción del molino. Lejos de ser para nosotros una dificultad, consideramos esto como una potencialidad que permi- tió a los estudiantes aprender o resignificar conceptos para que gracias a ellos las decisiones a tomar fueran fundadas.

Contar con un plano acabado, listo para ser utilizado, hubiera hecho que los estudiantes limiten sus acciones al cumplimiento de las indicaciones del plano. Es precisamente la ausencia de una guía acabada a seguir, la falta de un plano detallado, lo que provocó y permitió que los estudiantes tuvieran que reunirse a investigar.

Fue entonces la necesidad de tomar decisiones fundadas lo que motivó el aprendizaje y resignificación de conocimientos ya adquiridos. Entendemos que esto resulta fundamental para un funcionamiento didáctico de este tipo de proyectos. Volveremos sobre estos aspectos más adelante.

\section{El trabajo colectivo}

Otro aspecto que nos motivó fue el del trabajo colectivo de los estudiantes. Y por razones similares expuestas para los conocimientos, entendemos que rara vez un problema real se limita a una resolución individual. Consideramos que en la medida en que la complejidad aumenta, es indispensable el trabajo colectivo para su resolución. es por ello que nos interesamos en este proyecto a favorecer el trabajo en conjunto de los estudiantes, para que las potencialidades y dificultades de trabajar en equipo puedan ser discutidas en clase y así identificar aspectos a mejorar para futuros trabajos colectivos.

\section{El vínculo entre teoría y práctica}

Otro eje que nos interesó tratar fue el de la reunión de saberes denominados como teóricos y prácticos. Entendemos que los saberes o conocimientos necesarios para abordar problemas de la vida real requieren 
no solo de los formalizados bajo disciplinas tales como Matemática, Física o Estadística, sino que también son necesarios otros conocimientos vinculados a oficios o profesiones, saberes que en general no están formalizados (ni valorizados) en este tipo de carreras pero que resultan fundamentales a la hora de un trabajo concreto.

En efecto, esta integración no sólo fue requerida sino también reconocida y valorada por los estudiantes y profesores. Para la construcción e instalación del molino fueron necesarios entonces los conocimientos dichos teóricos que guiaron las acciones pero también los llamados prácticos que permitieron ejecutarlas y así concretar las respectivas construcciones. El proyecto facilitó también la integración en este sentido. Presentaremos una síntesis de nuestro esquema didáctico.

\section{Esquema didáctico}

Como comentamos anteriormente, el proyecto de construcción e instalación del molino fue considerado por nosotros como un marco que no solo motivaba a los estudiantes sino que también daba sentido a los aprendizajes del curso.

Desde el punto de vista didáctico, podemos decir que el proyecto resultó ser un eje cronológico y un marco de significación donde los conceptos eran convocados a los fines de resolver cuestiones ligadas a la fabricación e instalación del molino. Intentaremos resumir estas dos dimensiones interrelacionadas (cronológica y significación).

\section{Cronología y niveles de significación}

La cronología de aparición de un concepto no respondía tanto a razones epistemológicas propias a la disciplina de refe- rencia preestablecidas por un programa, por ejemplo de matemática, física o estadística, sino que ella estaba principalmente basada en otra lógica, la del proyecto. Así, los conocimientos en principio emergían en el momento en que eran necesarios para la resolución de alguna problemática referida a la construcción e instalación del molino.

A su vez, podemos decir que había dos niveles de significación para los conceptos. Aquellos conceptos que eran requeridos explícitamente por el proyecto, se encontraban en el nivel de significación directo. Por su parte, aquellos que eran necesarios para poder entender $y / u$ operar los del nivel de significación directo estaban en un nivel que llamamos indirecto.

Los conocimientos del nivel de significación directo respondían a una lógica de demanda del proyecto, los de nivel de significación indirecto lo hacían por necesidades epistemológicas. Por ejemplo, un concepto de nivel de significación directo fue el de función coseno, que permitía conocer el área de contacto al viento para así estimar la potencia teórica disponible en el molino. A ese concepto se le asociaba un conjunto de conceptos que eran necesarios, sea recordar sea aprender, para poder comprender el concepto requerido directamente por el proyecto. Tal fue el caso del estudio de funciones trigonométricas, su representación gráfica, suma de funciones, período, teorema de pitágoras, propiedades de ángulos entre rectas paralelas, etc.

Nos parece interesante remarcar que los niveles de profundización de los niveles de significación directo e indirecto son regulables en función del nivel de detalle que se desee y se pueda trabajar con los estudiantes. Podemos decir entonces que un proyec- 
to de este tipo permite trabajar un número muy importante de temas en función de cuánto se quiera y pueda profundizar en cada uno de los niveles comentados. Hemos podido comprobar que el interés que los alumnos manifiestan y el sentido que le dan a los conceptos en el nivel de significación indirecto no se ve fuertemente degradado, por lo que los estudiantes continúan trabajando en esos niveles ya más epistemológicos motivados por el sentido que proviene del nivel de significación directo.

Este tema está relacionado con uno que nos parece fundamental, el llamado motivacional, esta vez, como motor para los estudiantes. Suele ocurrir que los docentes nos lamentemos que nuestros estudiantes estudian sólo en proximidad de sus exá- menes y que su principal interés es el de aprobar la materia. Aquí, dado que los conocimientos encuentran un sentido o significación más allá del contexto de la clase , los estudiantes se ven motivados a trabajar.

En este tipo de propuestas, el compromiso de los estudiantes se desplaza de los centros convencionales de interés (exámenes, notas, etc.) y se orienta hacia, en este caso, el compromiso asumido para con la pobladora de construirle el molino para resolver su problema de agua.

Incluso el compromiso para con el profesor pasa a un segundo plano. En efecto, hemos observado cómo para los estudiantes y de manera progresiva, el interés por aprobar la materia retrocede como tema

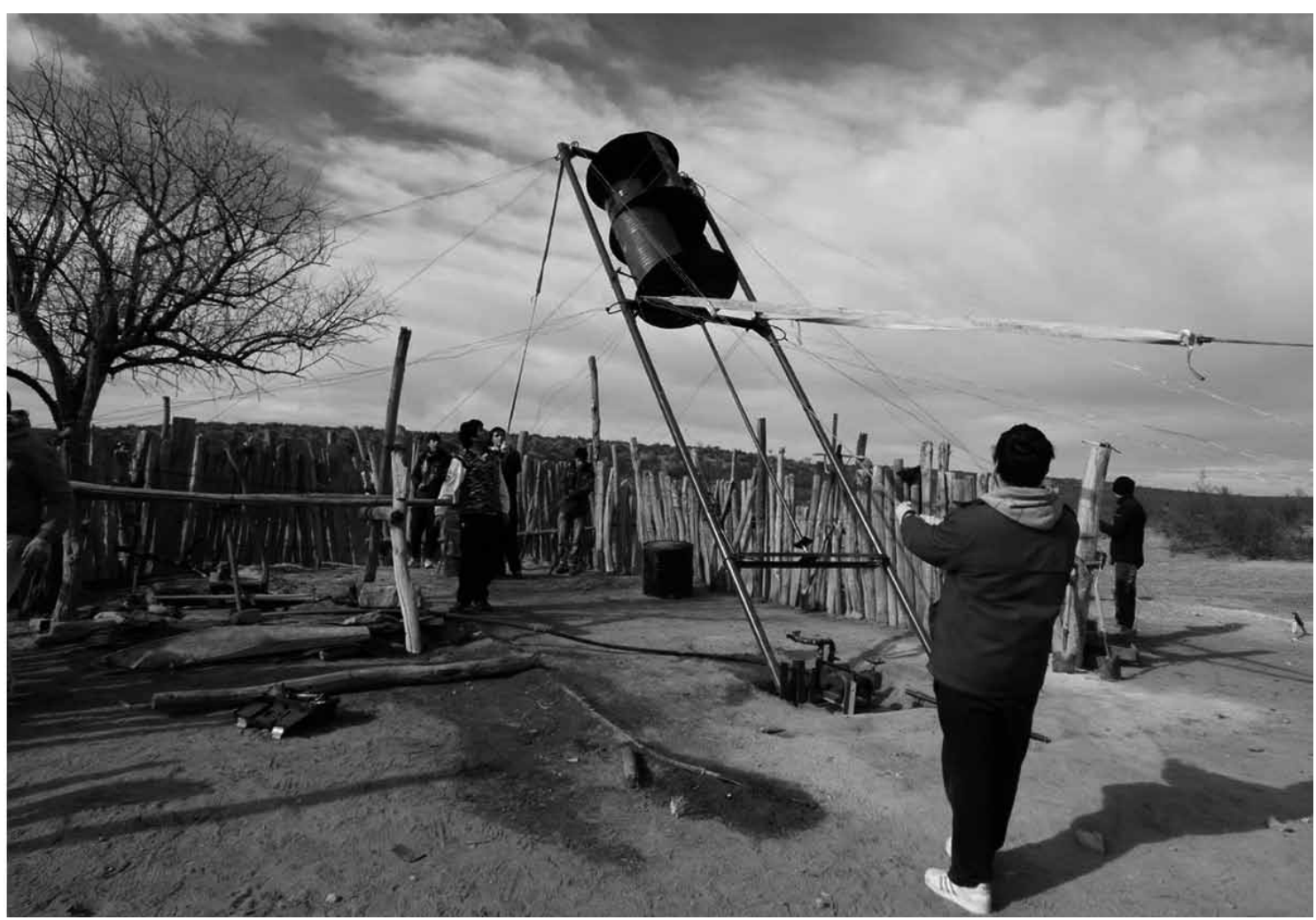

Figura 2. Montaje del Molino

de interés y cobra protagonismo el cum- beneficiario, en nuestro caso, Yolanda. plir con el compromiso asumido con el 


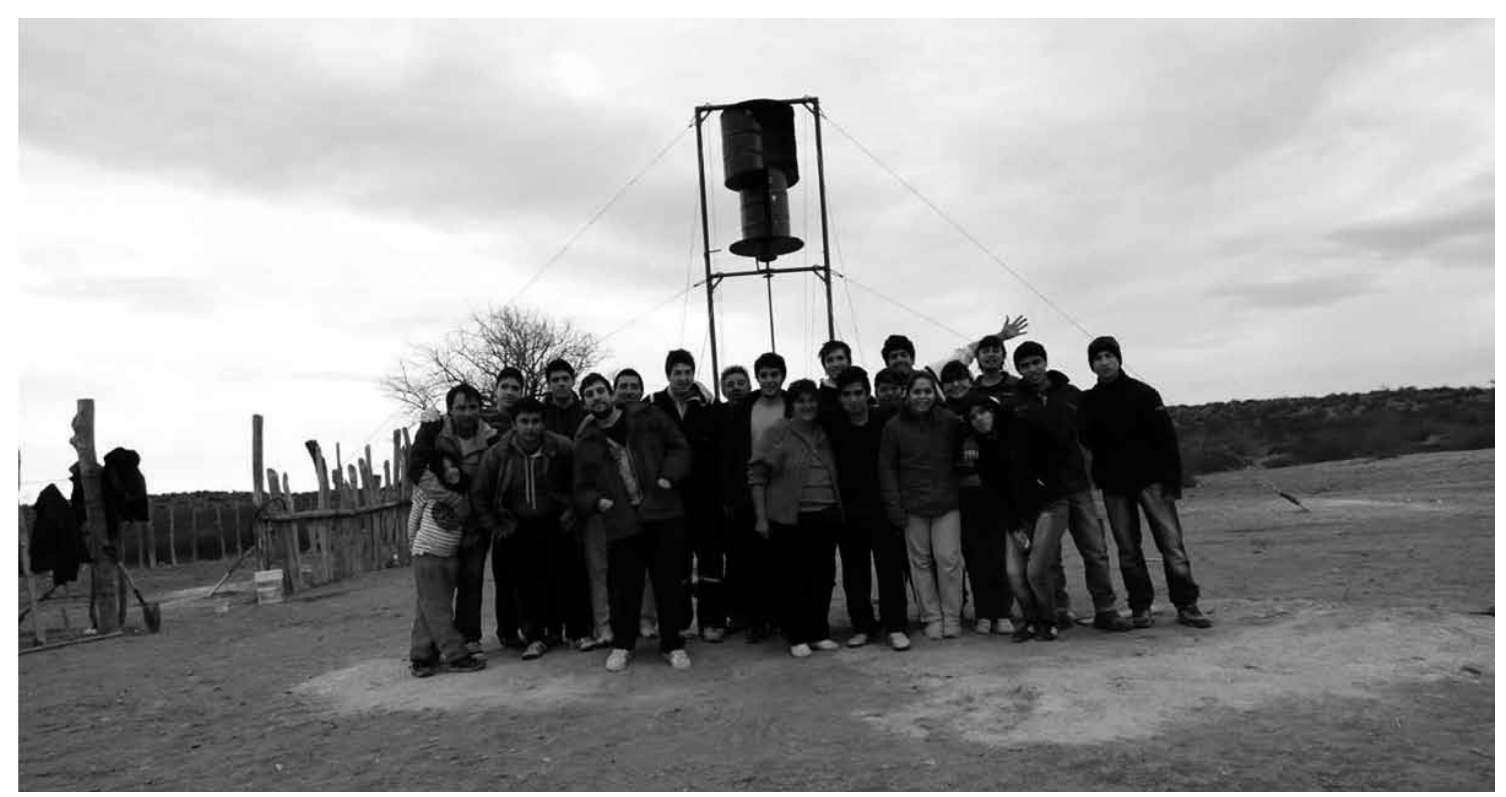

Figura 3. Montaje del Molino

Entendemos que en general este tipo de compromiso resulta entonces no solo productivo sino también sostenible. De hecho fue la fuerte motivación proveniente del compromiso para con la pobladora lo que permitió el trabajo extraclase necesario a la concreción del proyecto.

En efecto, la Tecnicatura en Mantenimiento Industrial no cuenta con talleres propios, por lo que las tareas de construcción de las partes y ensamblado final fueron realizadas en los hogares de los estudiantes. Otro ejemplo que ilustra el compromiso de los estudiantes es el momento de la instalación del molino. Fue en sus vacaciones de invierno y luego de dos intensos días de trabajo que juntos a los estudiantes logramos instalar el molino en el puesto de Yolanda.

Las figuras 2 y 3 muestras momentos de la instalación. Cabe acotar que en el puesto de Yolanda se carece de electricidad, agua, gas y teléfono. Para el montaje, debimos prever todos los elementos y herramientas necesarias, entre ellos un grupo electrógeno.

\section{La temporalidad}

Consideramos que la significación y el compromiso manifestado por los estudiantes con este tipo de trabajos están relacionados con lo que podríamos llamar la temporalidad. En otras palabras, los conceptos y métodos estudiados tienen un sentido en el presente (y admitimos que también para el futuro). Un presente de análisis y también de acción, que juntos permiten una intervención en su comunidad basada en argumentos provenientes de saberes aprendidos en su formación.

Como docentes solemos justificar a nuestros estudiantes que lo que están aprendiendo les servirá en un futuro y más allá que pueda ser cierto o no, eso puede vivirse como un tiempo de espera, de latencia, o de inversión para un futuro en el mejor de los casos.

Con este tipo de proyectos, el aprendizaje seve motivado por otra temporalidad, ya nola dela espera sinola delaacción en el presente. 
Este tipo de propuestas permiten vivenciar a los estudiantes el real uso de los saberes. Ellos permiten que nuestras acciones sean mejor fundadas, ganando así en seguridad, en economía y bienestar. En otras palabras, este tipo de propuesta pone no en hipótesis sino en actos la utilidad de los saberes aprendidos. Presentaremos algunas primeras reflexiones a modo de cierre.

\section{Primeras reflexiones}

Entendemos que los conceptos de temporalidad y motivación son importantes en este tipo de trabajos. Aquí, el conocimiento se vive como convocado para el presente. Intervenir en el presente racionalmente no nos parece un aspecto menor para la formación de nuestros estudiantes. Es un mensaje por medio de la acción misma que tiene implicancias en dos direcciones interrelacionadas: en su rol como miembro de una comunidad y en su rol como profesional.

En efecto, los mensajes son varios, por un ladoel conocimiento tiene una función colectiva,él sirve no solo para la mejora individual de quien lo posee sino que permite su intervención real y directa hacia su comunidad.

En lo que respecta a lo profesional, este tipo de trabajos ilustra para los estudiantes y para la comunidad, que los saberes resultan más provecho- sos que las soluciones intuitivas, aproximadas o realizadas por ensayo y error.

En este sentido, este tipo de trabajos vincula las necesidades reales con sus posibles soluciones. Este vínculo no siempre es trabajado en las formaciones y creemos es una de las causas del por qué muchos de los conocimientos adquiridos en las formaciones iniciales no son luego aplicados en la vida profesional. Tal es el caso por ejemplo de estadística donde existen en las empresas un número importante de datos disponibles que no son aprovechados para optimizar una solución a un problema dado. Entendemos que este tipo de trabajos muestra factualmente por un lado que los saberes son herramientas optimales a los problemas a los que nos vemos confrontados y por el otro, que uno puede no solamente ser consumidor sino también constructor de soluciones.

En lo que respecta a nuestra experiencia personal, podemos decir que el trabajo a motivado la integración entre profesores, no solo de la Tecnicatura en cuestión sino también con otras formaciones de la Universidad. En efecto, estamos elaborando un nuevo proyecto de construcción de un molino savonius donde participan colegas de otras materias de la carrera (mantenimiento industrial, Física, etc) y colegas de Geología que contribuirán para los estudios de suelo y agua. 